\title{
Distributed Formation Control without a Global Reference Frame
}

\author{
Eduardo Montijano, Dingjiang Zhou, Mac Schwager and Carlos Sagues
}

\begin{abstract}
This paper presents a decentralized controller to drive a team of agents to reach a desired formation in the absence of a global reference frame. Each agent is able to measure its relative position and orientation with respect to its neighbors. The different orientations imply that the relative positions between pairs of agents are sensed differently for each agent. In order to reach the desired configuration, the agents run two simultaneous consensus controllers, one to control their relative orientations, and another for their relative positions. The convergence to the desired configuration is shown by comparing the system with time-varying orientations with the equivalent approach with fixed rotations, showing that their difference vanishes as time goes to infinity. While the analysis in the paper is performed in a 2-dimensional space with orientations belonging to $S O(2)$, our approach can be extended to handle 3 dimensions and orientations in $S O(3)$. Simulation results, as well as hardware experiments with two quadrotor UAVs, corroborate the theoretical findings of the paper.
\end{abstract}

\section{INTRODUCTION}

Formation control is one of the cornerstone problems in decentralized control. Many multi-agent tasks require the agents to reach and maintain a given formation. In some applications all the agents need to travel with the same orientation in order to maintain the desired formation, for example for formation flying in fixed wing UAVs [1], or for some cooperative sensing scenarios [2]. In other applications agents are required to keep a formation with different orientations, such as aerial manipulation [3], environmental monitoring [4], or distributed escorting [5]. This need to maintain relative orientations as well as positions motivates the work in this paper.

Among existing formation control approaches, we can distinguish between leader-follower strategies and solutions based on nearest neighbor rules. Solutions of the first kind allow each agent to specify its movement depending only on a particular "leader" agent within the formation. Examples of controllers of this type have been presented using vision [6], considering relative bearing measurements [7], and including obstacle avoidance [8], to name a few. On the other hand, nearest-neighbor approaches let each agent design its motion using locally available information [9] from nearby agents. Nearest neighbor approaches frequently

E. Montijano is with Centro Universitario de la Defensa (CUD) and Instituto de Investigación en Ingeniería de Aragón (I3A), Zaragoza, Spain. emonti@unizar.es

D. Zhou and M. Schwager are with Department of Mechanical Engineering, Division of Systems Engineering, Boston University, United States. zdjatbu@gmail. com and schwagerabu . edu

C. Sagues is with Departamento de Informática e Ingeniería de Sistemas - Instituto de Investigación en Ingeniería de Aragón (I3A), Universidad de Zaragoza, Spain. csagues@unizar.es

This work was supported by the project DPI2012-32100. depend on a consensus-type control algorithm, and they have the advantage that they are robust to a broad range of interaction topologies between the team of agents.

Another important issue in the formation control problem is the specification of the desired configuration. The most common way of defining the formation is in terms of the relative information between pairs of agents. Depending on the sensors onboard them some approaches work with relative bearings [10], [11], relative distances [12], [13], or relative positions [14], [15]. However, a constant assumption in all of these approaches is the presence of a common rotation reference frame. In some cases it is because agents are modeled as $d$-dimensional points without orientations whereas in others it is assumed that there exist a previous agreement about a common rotation frame.

The difficulty with controlling orientation as well as position is that the orientation matrix appears as a multiplicative nonlinearity in the position dynamics of the agents. Hence the system dynamics in this case are nonlinear. We present a new consensus-based formation controller that is proven to drive the agents to the desired formation despite this nonlinearity. While consensus-based controllers have been deeply studied for positions [14], [15] and rotations [16], [17] independently, to the best of our knowledge there are no results analyzing the non-linear system that describes the evolution of the positions of the agents due to the variation of the rotation frames. Thus, the chief novelty in our problem lies in controlling both relative position and orientation for the agents at the same time.

In this paper we show that, with the proposed controller, the dynamics have a back-stepping structure that allows the orientation consensus to be proven first. Then the position consensus can be analyzed with the rotation matrix treated as a time-varying term that approaches a known limit. We prove that the coupled positions and orientations converge to a desired relative configuration. Additionally, while the analysis is done considering agents moving in $S E(2)$, the proposed control scheme is also well designed to work on $S E(3)$. To conclude, we also validate our proposal with simulations and in hardware experiments with two quadrotor UAVs.

The remainder of the paper is organized as follows: In Section II we introduce the notation of the paper and provide a formal definition of the problem we solve. Section III discusses the position controller when the agents have constant, but different rotation frames. The general case, with varying rotation frames is studied in Section IV. Simulation results are shown in Section V. Finally, Section VI presents the conclusions of the work and future lines of research. 


\section{Notation And Problem SetuP}

In the paper we consider a team of $N$ agents labeled by $\mathcal{V}=\{1, \ldots, N\}$. We let $\mathcal{F}_{\mathrm{w}}$ be some fixed (unknown) reference frame for the agents and $\mathcal{F}_{i}$ be the frame of agent $i$. The state of the agent $i$ expressed in $\mathcal{F}_{\mathrm{w}}$ is defined by its position, $\mathbf{p}_{i}=\left[x_{i}, y_{i}\right]^{T}$, and orientation, $\psi_{i}$. We denote by $\mathbf{R}_{i}$ the rotation matrix associated to $\psi_{i}$ :

$$
\mathbf{R}_{i}=\left(\begin{array}{cc}
\cos \left(\psi_{i}\right) & -\sin \left(\psi_{i}\right) \\
\sin \left(\psi_{i}\right) & \cos \left(\psi_{i}\right)
\end{array}\right) .
$$

Each agent is able to move with holonomic differential dynamics with respect to its own frame. Expressed in the world frame this is:

$$
\dot{\mathbf{p}}_{i}=\mathbf{R}_{i} \mathbf{v}_{i}
$$

with $\mathbf{v}_{i}=\left[v_{x_{i}}, v_{y_{i}}\right]^{T}$ the linear velocity in the local $\mathrm{x}$ and $\mathrm{y}$ directions and

$$
\dot{\psi}_{i}=w_{i}
$$

with $w_{i}$ the angular velocity. Under the dynamics described in (2), the time derivative of the rotation matrix, $\mathbf{R}_{i}$, is equal to

$$
\dot{\mathbf{R}}_{i}=\mathbf{S}_{i} \mathbf{R}_{i}
$$

with $\mathbf{S}_{i}$ the skew-symmetric matrix associated to $w_{i}$,

$$
\mathbf{S}_{i}=\left(\begin{array}{cc}
0 & -w_{i} \\
w_{i} & 0
\end{array}\right)
$$

We let $\mathbf{p}=\left[\mathbf{p}_{1}^{T}, \ldots, \mathbf{p}_{N}^{T}\right]^{T}$ denote the concatenation of the positions of all the agents and $\boldsymbol{\psi}=\left[\psi_{1}, \ldots, \psi_{N}\right]^{T}$ the concatenation of all the orientations.

Given two agents, we denote by $\mathbf{p}_{i j}$ and $\psi_{i j}$ the relative position and orientation of agent $j$ measured in the frame $\mathcal{F}_{i}$. The relative position between the agents is described by

$$
\mathbf{p}_{i j}=\left[x_{i j}, y_{i j}\right]^{T}=\mathbf{R}_{i}^{T}\left(\mathbf{p}_{j}-\mathbf{p}_{i}\right),
$$

whereas the relative rotation is, on a general instance, equal to the difference between the two rotations, i.e.,

$$
\psi_{i j}=\psi_{j}-\psi_{i}
$$

Additionally $\mathbf{R}_{i j}=\mathbf{R}_{i}^{T} \mathbf{R}_{j}$.

The objective of the agents is to end up in a configuration defined by the desired relative positions and orientations, $\mathbf{p}_{i j}^{*}$ and $\psi_{i j}^{*}$, between them.

Assumption 2.1: (Realizable configuration). The desired configuration can be achieved by the team, in the sense that all the relative positions and orientations are consistent with each other. Formally speaking, it must hold that

$$
\mathbf{p}_{i j}^{*}=\mathbf{p}_{i k}^{*}+\mathbf{R}_{i k}^{*} \mathbf{p}_{k j}^{*} \text { and } \psi_{i j}^{*}=\psi_{i k}^{*}+\psi_{k j}^{*},
$$

for all $i, j, k \in \mathcal{V}$, with $\mathbf{R}_{i k}^{*}$ the rotation matrix defined by the angle $\psi_{i k}^{*}$.

Note that there are infinite sets of positions in $\mathcal{F}_{\mathrm{w}}$ for the agents such that they satisfy (5), all of them related by some rotation and translation. Also note that in general $\mathbf{p}_{i j} \neq-\mathbf{p}_{j i}$.
Let $\mathcal{G}=(\mathcal{V}, \mathcal{E})$ be the interaction graph between the agents. The nodes in the graph, $\mathcal{V}$, are associated to the different agents, whereas the edges, $(i, j) \in \mathcal{E}$, represent the availability of the relative position and orientation of agent $j,\left[\mathbf{p}_{i j}, \psi_{i j}\right]$, to agent $i$. The set of neighbors of agent $i$ is defined as the set of agents perceived by agent $i$, i.e., $\mathcal{N}_{i}=\{j \in \mathcal{V} \mid(i, j) \in \mathcal{E}\}$, and $\left|\mathcal{N}_{i}\right|$ its cardinality. Let $\mathbf{L}=\left[l_{i j}\right]$ be the Laplacian matrix associated to $\mathcal{G}$ :

$$
l_{i j}= \begin{cases}\left|\mathcal{N}_{i}\right| & \text { if } i=j \\ -1 & \text { if }(i, j) \in \mathcal{E} \text { and } i \neq j . \\ 0 & \text { otherwise }\end{cases}
$$

Assumption 2.2: (Connected, undirected graph). The interaction graph, $\mathcal{G}$, is connected and undirected, i.e., there exist a path joining any two nodes and $(i, j) \in \mathcal{E} \Leftrightarrow(j, i) \in$ $\mathcal{E}$.

It is well known that under this assumption $\mathbf{L}$ has one eigenvalue, $\lambda_{1}=0$, with associated right (and left) eigenvector $\mathbf{1}_{N}=[1, \ldots, 1]^{T} \in \mathbb{R}^{N}$, and the rest of eigenvalues are all strictly positive. Along the paper, we let $\ell_{i}$ denote the $i^{\text {th }}$ row of the Laplacian matrix. Additionally, in the paper $\mathbf{I}_{k}, k \in \mathbb{N}$, denotes the identity matrix of dimension $k \times k$. To conclude, the Kronecker product between two matrices is represented by $\mathbf{A} \otimes \mathbf{B}$.

\section{Formation CONTROL WITH FIXED Rotation} FRAMES

Let us start by assuming that the robots have fixed, but not necessarily equal, rotation frames, that satisfy the following assumption:

Assumption 3.1: (Fixed desired orientations). The initial orientations of the agents are such that $\psi_{i j}=\psi_{i j}^{*}$ for all $i, j \in \mathcal{V}$.

Consequently, in this scenario, $\mathbf{R}_{i j}=\mathbf{R}_{i j}^{*}$ and $w_{i}=0$ for all $i, j \in \mathcal{V}$. The analysis of this simplified case will help us to demonstrate in the next section the convergence to the desired formation pattern of the general case with timevarying rotations.

The controller used by the agents is a standard consensus controller of the form:

$$
\mathbf{v}_{i}=\sum_{j \in \mathcal{N}_{i}}\left(\mathbf{p}_{i j}-\mathbf{p}_{i j}^{*}\right) .
$$

The following lemma will be useful for the analysis of both, the fixed and the time-varying rotations cases.

Lemma 3.2: (Eigenvalues of the "relative" Laplacian). Under Assumption 2.2 (Connected, undirected graph), the matrices $\mathbf{L}$ and $\mathbf{L}-\mathbf{1}_{N} \ell_{1}$ have exactly the same eigenvalues.

Proof: Since $\mathbf{L}$ is symmetric, it is diagonalizable. Thus, we can find an orthogonal matrix $\mathbf{P}$, such that $\mathbf{P}^{-1}=\mathbf{P}^{T}$ and

$$
\mathbf{P}^{T} \mathbf{L P}=\boldsymbol{\Lambda}
$$

with $\boldsymbol{\Lambda}=\operatorname{diag}\left(\lambda_{1}=0, \lambda_{2}, \ldots, \lambda_{N}\right)$ the diagonal matrix containing all the eigenvalues of $\mathbf{L}$. Additionally, note that the columns of $\mathbf{P}$ contain the set of (normalized) eigenvectors of $\mathbf{L}$, with the special case of the first column, equal to 
$\mathbf{1}_{N} / N$. Now, if we multiply $\mathbf{L}-\mathbf{1}_{N} \ell_{1}$ by $\mathbf{P}^{T}$ and $\mathbf{P}$ on the left and right respectively, we get:

$$
\mathbf{P}^{T}\left(\mathbf{L}-\mathbf{1}_{N} \ell_{1}\right) \mathbf{P}=\boldsymbol{\Lambda}-\mathbf{P}^{T} \mathbf{1}_{N} \ell_{1} \mathbf{P} .
$$

Noting that $\ell_{1} \mathbf{1}_{N}=0$

$$
\ell_{1} \mathbf{P}=\left(0, \lambda_{2} \gamma_{12}, \ldots, \lambda_{N} \gamma_{1 N}\right)
$$

with $\gamma_{1 i}$ the first component of the $i^{\text {th }}$ eigenvector of $\mathbf{L}$, and,

$$
\mathbf{P}^{T} \mathbf{1}_{N}=(1,0, \ldots, 0)^{T} .
$$

Therefore $\boldsymbol{\Lambda}-\mathbf{P}^{T} \mathbf{1}_{N} \ell_{1} \mathbf{P}$ is an upper triangular matrix, with the same elements in the diagonal as $\boldsymbol{\Lambda}$. Recalling that the matrix transformation given by $\mathbf{P}^{T}$ and $\mathbf{P}$ preserves the eigenvalues, we conclude that the matrix $\mathbf{L}-\mathbf{1}_{N} \ell_{1}$ has the same eigenvalues as the matrix $\mathbf{L}$.

Remark 3.3: (Eigenvectors of the "relative" Laplacian). Although the eigenvalues of $\mathbf{L}$ and $\mathbf{L}-\mathbf{1}_{N} \ell_{1}$ are the same, the eigenvectors of the two matrices are not necessarily equal. In fact, while the right eigenvector of $\mathbf{L}-\mathbf{1}_{N} \ell_{1}$ associated to $\lambda_{1}$ is the same as the one for $\mathbf{L}$, i.e., $\mathbf{1}_{N}$, the left eigenvector of the former matrix associated to $\lambda_{1}$ is $\zeta_{1}=(1,0, \ldots, 0)^{T}$, which is different than $\mathbf{1}_{N}$. These eigenvectors will be used later in the analysis.

Proposition 3.4: (Coonvergence with fixed rotation frames). Let Assumptions 2.1 (Realizable configuration), 2.2 (Connected, undirected graph) and 3.1 (Fixed desired orientations) be true. Then, using the controller in (6) the positions of the agents evolve in such a way that

$$
\lim _{t \rightarrow \infty} \mathbf{p}_{i j}=\mathbf{p}_{i j}^{*}, \forall i, j \in \mathcal{V} \text {. }
$$

Proof: Let us define the change of variables

$$
\mathbf{q}_{i}=\mathbf{p}_{1 i}-\mathbf{p}_{1 i}^{*}
$$

Using (3) and (5) and Assumption 3.1

$$
\mathbf{p}_{i j}=\mathbf{R}_{i 1}^{*}\left(\mathbf{p}_{1 j}-\mathbf{p}_{1 i}\right)
$$

and similarly $\mathbf{p}_{i j}^{*}=\mathbf{R}_{i 1}^{*}\left(\mathbf{p}_{1 j}^{*}-\mathbf{p}_{1 i}^{*}\right)$. Note that in the (unknown) global frame $\mathbf{R}_{i 1}^{*}=\mathbf{R}_{i}^{T} \mathbf{R}_{1}$ for some fixed $\mathbf{R}_{i}$ and $\mathbf{R}_{1}$, which are also the rotation matrices that appear in (1). Thus

$$
\dot{\mathbf{p}}_{i}=\mathbf{R}_{1} \sum_{j \in \mathcal{N}_{i}}\left(\mathbf{q}_{j}-\mathbf{q}_{i}\right) .
$$

Differentiating (11),

$$
\begin{aligned}
\dot{\mathbf{q}}_{i} & =\mathbf{R}_{1}^{T}\left(\dot{\mathbf{p}}_{i}-\dot{\mathbf{p}}_{1}\right) \\
& =\sum_{j \in \mathcal{N}_{i}}\left(\mathbf{q}_{j}-\mathbf{q}_{i}\right)-\sum_{k \in \mathcal{N}_{1}}\left(\mathbf{q}_{k}-\mathbf{q}_{1}\right),
\end{aligned}
$$

which, denoting $\mathbf{q}=\left(\mathbf{q}_{1}, \ldots, \mathbf{q}_{N}\right)^{T}$, yields in matricial form

$$
\dot{\mathbf{q}}=-\left[\left(\mathbf{L}-\mathbf{1}_{N} \ell_{1}\right) \otimes \mathbf{I}_{2}\right] \mathbf{q}
$$

By Lemma 3.2, the matrix $-\left(\mathbf{L}-\mathbf{1}_{N} \ell_{1}\right)$, has one eigenvalue equal to zero, and the rest of eigenvalues strictly negative. Thus

$$
\lim _{t \rightarrow \infty} \mathbf{q}=\left(\frac{\mathbf{1}_{N} \boldsymbol{\zeta}_{1}^{T}}{\boldsymbol{\zeta}_{1}^{T} \mathbf{1}_{N}} \otimes \mathbf{I}_{2}\right) \mathbf{q}(0)=\mathbf{1}_{N} \mathbf{q}_{1}(0),
$$

with $\boldsymbol{\zeta}_{1}=(1,0, \ldots, 0)^{T}$. However, note that $\mathbf{q}_{1}$ is always constant and equal to $\mathbf{0}$, and therefore $\mathbf{q} \rightarrow \mathbf{0}$. Consequently $\mathbf{p}_{1 i}$ tends to $\mathbf{p}_{1 i}^{*}$ for all $i$, and because of Assumption 2.1 the result is proved.

Remark 3.5: (Comparison with [14]) The controller in (6) is similar the one in [14], with the exception that it allows the agents to have different (but fixed) rotation frames. The analysis in the proof of Proposition 3.4 is also somewhat different to accommodate these different rotations. We will leverage this intermediate result to prove our main result with time-varying rotation frames in the next section.

\section{FORMATION CONTROL WITH VARYING ROTATION FRAMES}

In this section we analyze the full system when both the translation and the rotation evolve in the time.

Initially, we require to make an assumption about the initial orientations of the agents:

Assumption 4.1: (Relative initial orientations). The initial relative orientations satisfy

$$
\max _{i \in \mathcal{V}}\left(\psi_{1 i}-\psi_{1 i}^{*}\right)-\min _{i \in \mathcal{V}}\left(\psi_{1 j}-\psi_{1 j}^{*}\right)<\pi
$$

As in the previous section, we consider that the linear velocities of the agents are defined according to (6), whereas in order to reach the desired relative rotations, the agents execute the consensus controller:

$$
w_{i}=\sum_{j \in \mathcal{N}_{i}}\left(\psi_{i j}-\psi_{i j}^{*}\right) .
$$

Theorem 4.2: (Coonvergence with time-varying rotation frames) Let Assumptions 2.1 (Realizable configuration), 2.2 (Connected, undirected graph) and 4.1 (Relative initial orientations) be true. Then, using the controller in (6) and (18) the positions and orientations of the agents evolve in such a way that

$$
\lim _{t \rightarrow \infty} \mathbf{p}_{i j}=\mathbf{p}_{i j}^{*}, \text { and } \lim _{t \rightarrow \infty} \psi_{i j}=\psi_{i j}^{*},
$$

for all $i, j \in \mathcal{V}$.

Proof: According to the dynamics in (1) and (2), using the control inputs in (6) and (18) the evolution of the orientations is decoupled from the positions of the agents. This implies that we can analyze the orientations separately and study afterwards what happens with the positions of the agents with the time-varying rotation frames.

In order to prove the convergence of the orientations we define the change of variables $\theta_{i}=\psi_{1 i}-\psi_{1 i}^{*}$ and $\boldsymbol{\theta}=\left(\theta_{1}, \ldots, \theta_{N}\right)^{T}$. Note that the new variables are also orientations that belong to the manifold $S O(2)$. Assumption 4.1 ensures that all these new orientations are contained in a ball of radius $\pi$ with respect to some reference frame, a necessary condition in order to reach consensus when measuring relative orientations [16], [18].

After some manipulations, the dynamics of $\boldsymbol{\theta}$ can be expressed by

$$
\dot{\boldsymbol{\theta}}=-\left(\mathbf{L}-\mathbf{1}_{N} \ell_{1}\right) \boldsymbol{\theta}
$$


Using similar arguments as in Proposition 3.4 we reach

$$
\lim _{t \rightarrow \infty} \boldsymbol{\theta}=\frac{\mathbf{1}_{N} \boldsymbol{\zeta}_{1}^{T}}{\boldsymbol{\zeta}_{1}^{T} \mathbf{1}_{N}} \boldsymbol{\theta}(0)=\theta_{1}(0) \mathbf{1}_{N},
$$

and since $\theta_{1}=0$ for all $t, \psi_{1 i}$ converges to $\psi_{1 i}^{*}$ for all $i$. Finally, because of Assumption 2.1 we conclude that all the pairs of agents reach their desired relative rotations.

At this point we know that the rotations asymptotically converge to a constant value such that $\mathbf{R}_{i j}=\mathbf{R}_{i j}^{*}$ and $w_{i}=0$ for all the agents. We also know that controller (6) with constant rotations reaches the desired configuration. However, these two arguments do not guarantee that the agents will end up with the desired relative positions. A new analysis of the positions is required.

In order to simplify the notation, let us define

$$
\mathbf{b}_{i}^{*}=\sum_{j \in \mathcal{N}_{i}} \mathbf{p}_{i j}^{*}
$$

and $\mathbf{b}^{*}=\left[\mathbf{b}_{1}^{*}, \ldots, \mathbf{b}_{N}^{*}\right]^{T}$. The dynamics of the positions in the world frame can be expressed now by

$$
\dot{\mathbf{p}}=-\left(\mathbf{L} \otimes \mathbf{I}_{2}\right) \mathbf{p}-\operatorname{diag}\left(\mathbf{R}_{i}\right) \mathbf{b}^{*},
$$

with $\operatorname{diag}\left(\mathbf{R}_{i}\right)=\operatorname{diag}\left(\mathbf{R}_{1}, \ldots, \mathbf{R}_{N}\right) \in \mathbb{R}^{2 N \times 2 N}$ the block diagonal matrix defined by the $N$ rotational matrices of the agents in the (unknown) global frame. Note that, in terms of $\mathbf{p}$, this dynamics corresponds to a non-homogeneous linear time-variant system, because $\mathbf{R}_{i}$ changes with the time, but with a known evolution.

Let us consider the change of variables

$$
\mathbf{q}_{i}=\mathbf{p}_{1 i}=\mathbf{R}_{1}^{T}\left(\mathbf{p}_{i}-\mathbf{p}_{1}\right),
$$

that transforms all the positions relative to agent 1. Using (3) and (5)

$$
\begin{aligned}
\mathbf{p}_{i j} & =\mathbf{p}_{i 1}+\mathbf{R}_{i 1} \mathbf{p}_{1 j}=-\mathbf{R}_{i 1} \mathbf{p}_{1 i}+\mathbf{R}_{i 1} \mathbf{p}_{1 j} \\
& =\mathbf{R}_{i}^{T} \mathbf{R}_{1}\left(\mathbf{p}_{1 j}-\mathbf{p}_{1 i}\right) .
\end{aligned}
$$

Hence

$$
\begin{aligned}
\dot{\mathbf{p}}_{i} & =\mathbf{R}_{i} \sum_{j \in \mathcal{N}_{i}} \mathbf{p}_{i j}-\mathbf{R}_{i} \mathbf{b}_{i}^{*} \\
& =\mathbf{R}_{1} \sum_{j \in \mathcal{N}_{i}}\left(\mathbf{q}_{j}-\mathbf{q}_{i}\right)-\mathbf{R}_{i} \mathbf{b}_{i}^{*} .
\end{aligned}
$$

Differentiating (20) and using some algebra,

$$
\begin{aligned}
\dot{\mathbf{q}}_{i} & =\dot{\mathbf{R}}_{i}^{T}\left(\mathbf{p}_{i}-\mathbf{p}_{1}\right)+\mathbf{R}_{1}^{T}\left(\dot{\mathbf{p}}_{i}-\dot{\mathbf{p}}_{1}\right) \\
& =\mathbf{S}_{1}^{T} \mathbf{q}_{i}+\sum_{j \in \mathcal{N}_{i}}\left(\mathbf{q}_{j}-\mathbf{q}_{i}\right)-\mathbf{R}_{1}^{T} \mathbf{R}_{i} \mathbf{b}_{i}^{*}- \\
& -\sum_{k \in \mathcal{N}_{1}}\left(\mathbf{q}_{k}-\mathbf{q}_{1}\right)+\mathbf{b}_{1}^{*},
\end{aligned}
$$

and, piling the variables $\mathbf{q}=\left(\mathbf{q}_{1}, \ldots, \mathbf{q}_{N}\right)^{T}$,

$$
\begin{aligned}
\dot{\mathbf{q}} & =\left[\mathbf{I}_{N} \otimes \mathbf{S}_{1}^{T}-\left(\mathbf{L}-\mathbf{1}_{N} \ell_{1}\right) \otimes \mathbf{I}_{2}\right] \mathbf{q}- \\
& -\operatorname{diag}\left(\mathbf{R}_{1 i}\right) \mathbf{b}^{*}+\mathbf{b}_{1}^{*} \otimes \mathbf{1}_{N},
\end{aligned}
$$

with $\operatorname{diag}\left(\mathbf{R}_{1 i}\right)=\operatorname{diag}\left(\mathbf{R}_{11}, \ldots, \mathbf{R}_{1 N}\right) \in \mathbb{R}^{2 N \times 2 N}$ the block diagonal matrix defined by the $N$ relative rotational matrices of the agents with respect to agent 1 .
In order to analyze the new system, we are going to compare it with the time-invariant system described in section III with fixed rotations. To distinguish them, in the proof we will denote by $\overline{\mathbf{p}}$ the system with fixed rotations. Similarly, we let $\overline{\mathbf{q}}$ be the same change of variables as in (20), whose dynamics is

$$
\dot{\overline{\mathbf{q}}}=-\left[\left(\mathbf{L}-\mathbf{1}_{N} \ell_{1}\right) \otimes \mathbf{I}_{2}\right] \overline{\mathbf{q}}-\operatorname{diag}\left(\mathbf{R}_{1 i}^{*}\right) \mathbf{b}^{*}+\mathbf{b}_{1}^{*} \otimes \mathbf{1}_{N},
$$

with $\operatorname{diag}\left(\mathbf{R}_{1 i}^{*}\right)$ defined as $\operatorname{diag}\left(\mathbf{R}_{1 i}\right)$ with the desired relative rotations.

Let us denote

$$
\mathbf{e}=\mathbf{q}-\overline{\mathbf{q}},
$$

the difference between the two relative positions. Using (23) and (24), the evolution in the time of $\mathbf{e}$ is given by

$$
\dot{\mathbf{e}}=\mathbf{W e}+\boldsymbol{\beta},
$$

with

$$
\mathbf{W}=\left[\mathbf{I}_{N} \otimes \mathbf{S}_{1}^{T}-\left(\mathbf{L}-\mathbf{1}_{N} \ell_{1}\right) \otimes \mathbf{I}_{2}\right],
$$

and

$$
\boldsymbol{\beta}=\left(\mathbf{I}_{N} \otimes \mathbf{S}_{1}^{T}\right) \overline{\mathbf{q}}+\left(\operatorname{diag}\left(\mathbf{R}_{1 i}^{*}\right)-\operatorname{diag}\left(\mathbf{R}_{1 i}\right)\right) \mathbf{b}^{*} .
$$

Let us remark that even when e depends on $\overline{\mathbf{q}}$, the evolution of this variable is known, and for that reason it can also be put as a part of $\boldsymbol{\beta}$ (the same cannot be said about $\mathbf{q}$ because its evolution is still unknown).

The system in (26) is another non-autonomous linear time-varying system, because both $\mathbf{W}$ and $\boldsymbol{\beta}$ change with the time. In order to demonstrate that it converges to zero let us first consider the autonomous version of (26), i.e, $\boldsymbol{\beta}=0$ for all times. Consider the following candidate Lyapunov function $V=\mathbf{e}^{T} \mathbf{e}$, which is positive definite and has time derivative equal to $\dot{V}=2 \mathbf{e}^{T} \mathbf{W e}$. Replacing $\mathbf{W}$ by the expression in (27), since $\mathbf{S}_{1}^{T}$ is a skew symmetric matrix, $\mathbf{e}^{T}\left(\mathbf{I}_{N} \otimes \mathbf{S}_{1}^{T}\right) \mathbf{e}=0$ for any value of $w_{1}$. Recalling Lemma (3.2),

$$
2 \mathbf{e}^{T}\left(-\left(\mathbf{L}-\mathbf{1}_{N} \ell_{1}\right) \otimes \mathbf{I}_{2}\right) \mathbf{e} \leq-2 \lambda_{2}\|\mathbf{e}\|^{2},
$$

with $\lambda_{2}$ the second smallest eigenvalue of $\mathbf{L}$, which because of Assumption 2.2 is strictly positive. Then, the system (26) is input-to-state stable (Lemma 4.6 in [19]).

Additionally, it holds that $\lim _{t \rightarrow \infty} \boldsymbol{\beta}=\mathbf{0}$ because we know that $\lim _{t \rightarrow \infty} \mathbf{R}_{1 i}=\mathbf{R}_{1 i}^{*}$ for all $i$ and $\lim _{t \rightarrow \infty} w_{i}=$ $0 \Rightarrow \lim _{t \rightarrow \infty} \mathbf{S}_{i}^{T}=\mathbf{0}$. Therefore, using again [19] we conclude that e converges to zero. Consequently, for all $i$ it holds that

$$
\lim _{t \rightarrow \infty} \mathbf{q}_{i}=\lim _{t \rightarrow \infty} \overline{\mathbf{q}}_{i}=\mathbf{p}_{1 i}^{*},
$$

and by Assumption 2.1 we conclude that all the pairs of agents also reach their desired relative positions.

Remark 4.3: (Analysis in the local frame). Note that the part of the proof of Theorem 4.2 dealing with the positions cannot be analyzed in the global frame, that is, considering the difference between the variables $\mathbf{p}$ and $\overline{\mathbf{p}}$ instead of $\mathbf{q}$ and $\overline{\mathbf{q}}$ in eq. (25). In fact, the difference between these two variables does not necessarily go to zero, because the 



Fig. 1. Distributed formation control with time-varying rotation frames. The figure shows the desired configuration (left), the initial configuration (middle) and the trajectories with the final configuration of the agents (right).

evolution of the positions of the agents in $\mathcal{F}_{\mathrm{w}}$ is different for the two variables.

Remark 4.4: (Extension to time-varying topologies). Although we do not prove it in the paper, it should be noted that, considering that our approach is based on standard consensus-controllers, the team of agents will also be able to form in the desired pattern in a scenario with time-varying interaction topologies, given the standard requirements of bounded dwell time and periodic joint connectivity [9].

Remark 4.5: (Extension to three dimensions). The formulation used in the paper makes our approach easily extensible to $S E(3)$. The only requirement is to find an appropriate consensus controller that ensures convergence of the orientations of the agents in $S O(3)$ with relative measurements and without taking into account the relative positions. Fortunately, there are already existing works in the literature that present solutions to this problem, e.g., [18], [20]. In such case, if the agents use the position controller given in (6), the analysis presented here is still valid and thus, the convergence to the desired relative $3 D$ configuration.

\section{Simulations AND EXPERIMENTS}

In this section we validate the theoretical results of the paper with an illustrative example using a simulated environment as well as with hardware experiments with two quadrotor UAVs.

\section{A. Simulation Results}

In this section we demonstrate the behavior of our formation controller with an illustrative example carried out using MATLAB. We consider a team of $N=10$ mobile agents. We design the desired formation such that the agents are equally spaced around the perimeter of a circle, oriented so that they are all pointing outward from the circle. This is shown in the left plot of Fig. 1, where the configuration is shown in the world frame.

The initial configuration of the agents is depicted in the middle plot of Fig. 1, and is chosen such that the angle condition in Assumption 4.1 is satisfied. The trajectories of the positions as well as the final orientation of the agents obtained using the proposed controller can be seen in the right plot of Fig. 1. As proven in Theorem 4.2, we can observe that the team has reached the configuration with all the relative positions and orientations equal to the desired ones, despite each one having a different reference frame. Note that in the left plot of Fig. 1 we are using the world frame for simplicity in depicting the desired relative positions of the agents, but, as in fact happens, the final positions of the agents in the global frame (right plot of Fig. 1) are given by a rigid body transformantion than those of the left one. However, the relative positions and orientations between the agents are equal in both cases. Finally, the evolution of the orientation of the agents measured in the global frame is shown in Fig. 2.

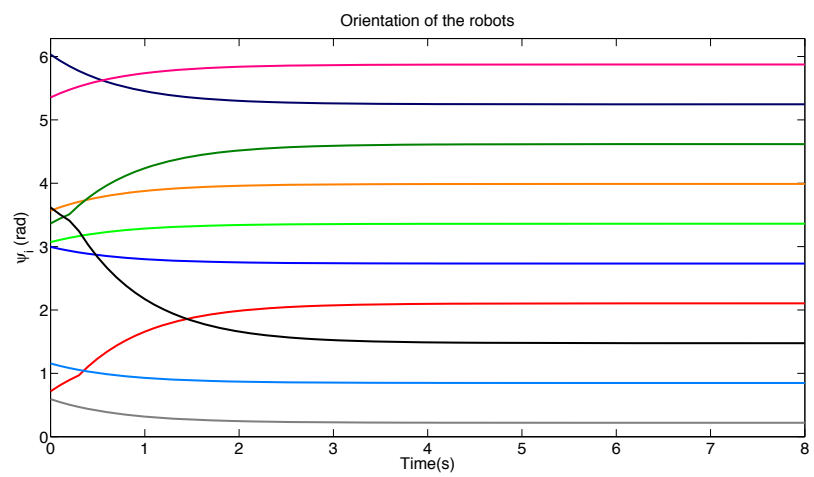

Fig. 2. Evolution of the orientation of the agents measured in $\mathcal{F}_{\mathrm{w}}$

\section{B. Hardware Experiments}

Here we present the results of an experiment with two quadrotor robots in a motion capture environment to verify the performance of the controller. The robots used in the experiments are KMel kQuad500 quadrotors (http://kmelrobotics.com/). The position and orientation of the quadrotors are observed by an OptiTrack motion capture system (NaturalPoint, Inc, http://www . naturalpoint.com/optitrack/) at an update rate $120 \mathrm{~Hz}$ and obtained by MATLAB via Java scripts reading UDP packets from the OptiTrack system.

The desired trajectory for each quadrotor is computed in MATLAB using the control law described in (6) and (18). This trajectory is implemented for the quadrotors using a differential-flatness based trajectory following controller, the 


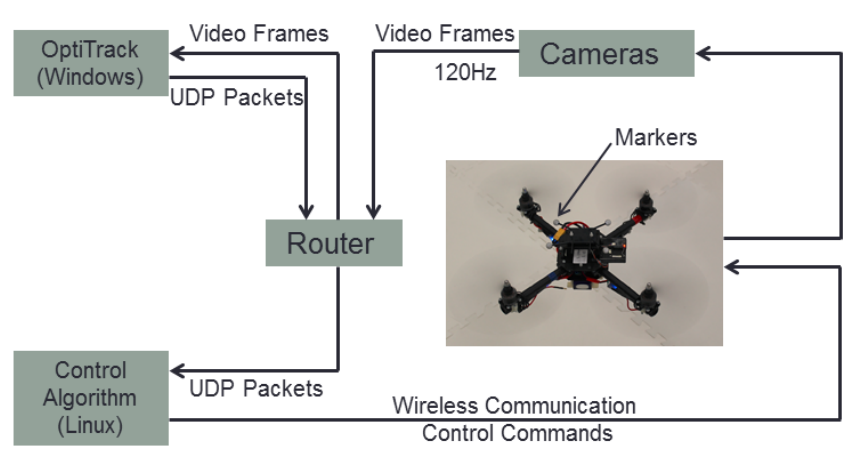

Fig. 3. System diagram.

details of which can be found in [21]. The desired angular and linear accelerations for realizing the desired trajectory are then sent over a ZigBee wireless link to the quadrotors to close the control loop. Furthermore, each quadrotor uses a low level on-board controller running at $500 \mathrm{~Hz}$ to achieve the desired angular and linear accelerations, using an onboard IMU for feedback. Figure 3 shows a diagram of this experimental setup.

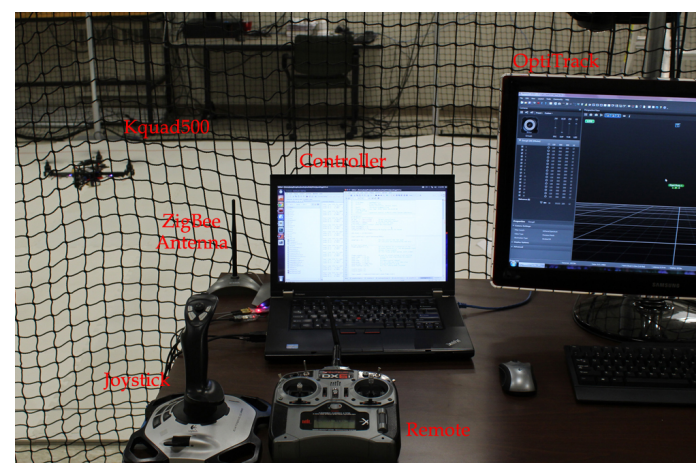

Fig. 4. Control station, with one Kquad500 flying in the arena.

Figure 4 shows the control station with the OptiTrack computer (Windows), Flight control computer (Ubuntu), a joystick (Logitech Extreme 3D pro), a Spectrum remote control unit, and the antenna used for autonomous flight control. The joystick and the remote control unit are used for manual flight and also for switching between the manual flight and autonomous flight.

The results of our experiment are shown in Fig. 5, with a composite photograph of the experiment on the top, and the corresponding MATLAB simulation on the bottom. One can see from the figure that the experimental results match the simulation well. The quadrotors change both their orientation and position in moving from their initial configuration (with the robots at the far left and right of the frame), to their final configuration (with the robots facing upward and downward at the top and bottom of the frame). The robots compute their control action using relative measurements in their own local reference frame, and they have no knowledge of a global reference frame. They reach a consensus on a global reference frame, while simultaneously achieving the desired configuration, as proven in Theorem 4.2. A movie of the

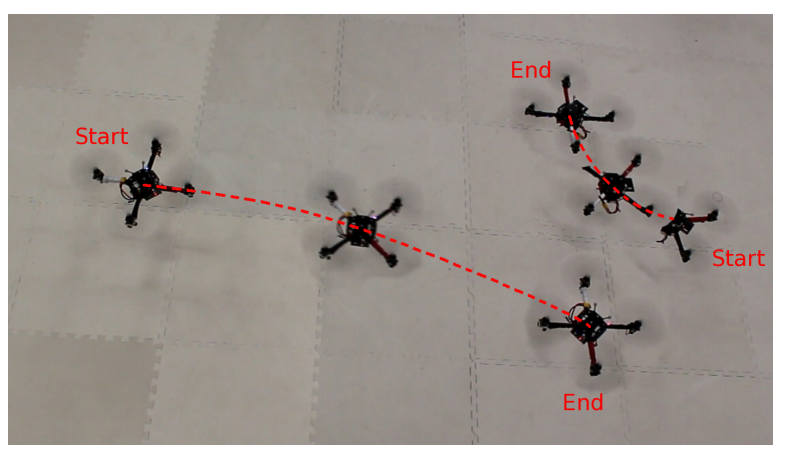

(a) Experiment

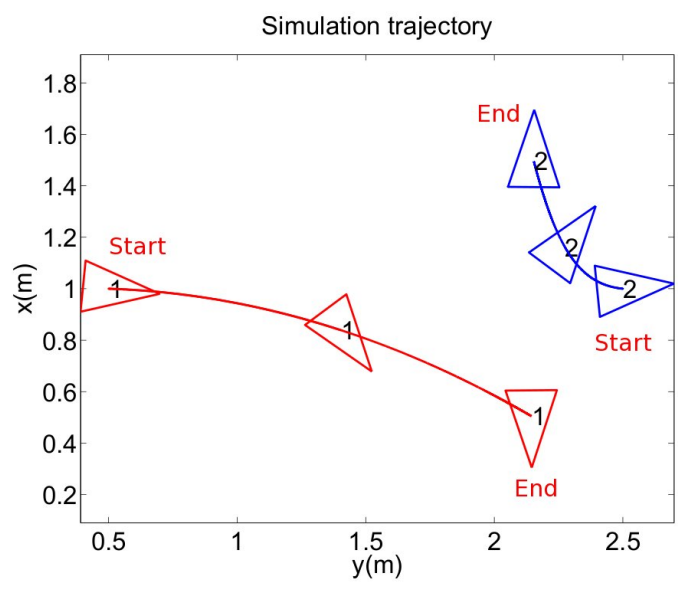

(b) Simulation

Fig. 5. This figure shows a composite photograph of the experiment on top, with the corresponding MATLAB simulation below it. The quadrotors start at the far left and right of the frame, and move to the bottom and top, respectively.

experiment can be found at http://people.bu.edu/ schwager/Movies/MontijanoACC13Movie.mov.

The time evolution of the relative positions of the two robots are shown in Fig. 6 and their relative orientations are shown in Fig. 7. The effects of the different rotation frames are clearly reflected in these plots. The final configuration for both robots is to have the other robot 1 meter behind them, with their orientations 180degs apart. We can observe that in the relative frames both ' $y$ ' coordinates converge to -1 , while both ' $\mathrm{x}$ ' coordinates converge to 0 . If they shared a common reference frame, the two 'y' coordinates would have to be $1 \mathrm{~m}$ and $-1 \mathrm{~m}$ to describe this formation. In general, the presence of time varying reference frames complicates the formation control problem because changes in rotation result in nonlinear changes in relative position. The controller in this paper is proven to converge to the desired formation despite this nonlinearity, as verified in the experiments.

\section{CONCLUSIONS}

In this paper we have presented a distributed consensus controller that drives a team of agents to reach a desired formation with specified relative positions and orientations in the absence of a global reference frame. Although the time-varying rotations make the system dynamics nonlinear, 



(a) Position of quad 2 from quad 1
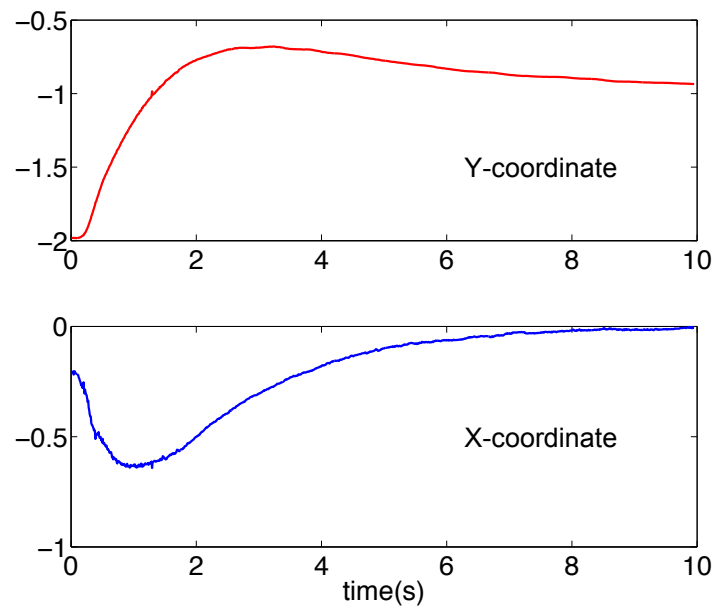

(b) Position of quad 1 from quad 2

Fig. 6. The $\mathrm{x}$ (blue) and $\mathrm{y}$ (red) positions of quadrotor 2 as seen from quadrotor 1 is shown in the top plots, and of quad 1 as seen from quad 2 is shown in the bottom plots. The final configuration has the quads back to back, separated by 1 meter, as shown in these plots.

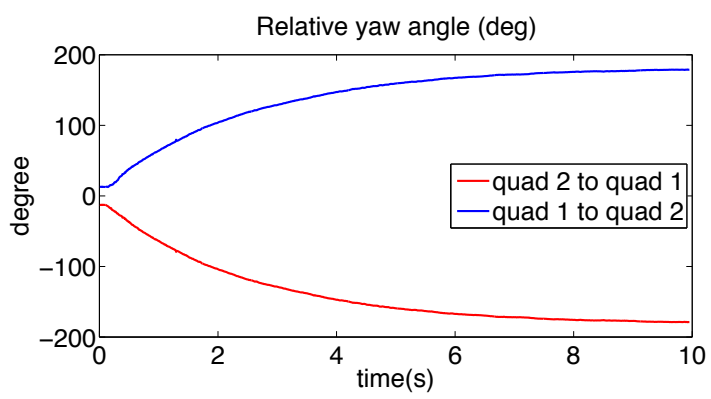

Fig. 7. The relative angles of the two quadrotors are shown here. Red shows the angle of quad 1 as seen from quad 2, and the blue curve shows quad 2 as seen from quad 1 . One can see that the robots converge to a configuration in which they are back-to back, separated by an angle of $180 \mathrm{deg}$.

the back-stepping structure of our approach has allowed us to separate the analysis in the rotations and the positions. The convergence to the desired configuration has been shown demonstrating that the difference between the system with time-varying orientations and an equivalent system with fixed rotations approaches zero in time. Additionally, the proposed structure makes the extension to 3 dimensions and orientations in $S O(3)$ straightforward, provided that the agents use an appropriate consensus controller for $S O(3)$. We have demonstrated the feasibility of the theoretical analysis in a simulated scenario and with a hardware implementation using two quadrotor UAVs. Current research is being devoted to the estimation of the relative positions on-board by means of computer vision.

\section{REFERENCES}

[1] R. Fierro, C. Belta, J. Desai, and V. Kumar. On controlling aircraft formations. In Proc. of the IEEE Conference on Decision and Control, pages 1065-1070, 2001.

[2] F. Zhang and N. E. Leonard. Cooperative filters and control for cooperative exploration. IEEE Transactions on Automatic Control, 55(3):650-663, March 2010.

[3] N. Michael, J. Fink, and V. Kumar. Cooperative manipulation and transportation with aerial robots. Autonomous Robots, 30(1):73-86, January 2011.

[4] M. Schwager, B. Julian, M. Angermann, and D. Rus. Eyes in the sky: Decentralized control for the deployment of robotic camera networks. Proceedings of the IEEE, 99(9):1541-1561, Sept 2011.

[5] Y. Lan, Z. Lin, M. Cao, and G. Yan. A distributed reconfigurable control law for escorting and patrolling missions using teams of unicycles. In IEEE Conference on Decision and Control, pages 54565461, December 2010.

[6] A. Das, R. Fierro, V. Kumar, J. Ostrowski, J. Spletzer, and C. J. Taylor. Vision based formation control of multiple robots. IEEE Transactions on Robotics and Automation, 18(5):813-825, Oct 2002.

[7] T. Gustavi and X. Hu. Observer-based leader-following formation control using onboard sensor information. IEEE Transactions on Robotics, 24(6):1457-1462, Dec 2008.

[8] P. Urcola and L. Montano. Cooperative robot team navigation strategies based on an environment model. In IEEE/RSJ International Conference on Intelligent Robots and Systems, pages 4577-4583, St. Louis, USA, October 2009.

[9] A. Jadbabaie, J. Lin, and A.S. Morse. Coordination of Groups of Mobile Autonomous Agents using Nearest Neighbor Rules. IEEE Transactions on Automatic Control, 48(6):988-1001, June 2003.

[10] M. Basiri, A. N. Bishop, and P. Jensfelt. Distributed control of triangular formations with angle-only constraints. Systems \& Control Letters, 59(1):147-154, Jan 2010.

[11] A. Franchi and P. R. Giordano. Decentralized control of parallel rigid formations with direction constraints and bearing measurements. In 51th IEEE International Conference on Decision and Control, pages 5310-5317, Dec 2012.

[12] M. Cao, C. Yu, and B. D.O. Anderson. Formation control using rangeonly measurements. Automatica, 47(4):776-781, April 2011.

[13] K-K Oh and H-S Ahn. Formation control of mobile agents based on inter-agent distance dynamics. Automatica, 47(10):2306-2312, Oct 2011.

[14] J. Cortés. Global and robust formation-shape stabilization of relative sensing networks. Automatica, 45(12):2754-2762, Dec 2009.

[15] W. Ren. Collective motion from consensus with cartesian coordinate coupling. IEEE Transactions on Automatic Control, 54(6):1330-1335, June 2009.

[16] N. Mostagh and A. Jadbabaie. Distributed geodesic control laws for flocking of nonholonomic agents. IEEE Transactions on Automatic Control, 52(4):681-686, 2007.

[17] E. Montijano, J. Thunberg, X. Hu, and C. Sagues. Epipolar visual servoing for multi-robot distributed consensus. IEEE Transactions on Robotics, 2013. To appear.

[18] J. Thunberg, E. Montijano, and X. Hu. Distributed attitude synchronization control. In 50th IEEE Conference on Decision and Control and European Control Conference, pages 1962-1967, December 2011.

[19] H. K. Khalil. Nonlinear Systems. Third Edition. Prentice Hall, 2002.

[20] A. Sarlette and R. Sepulchre. Consensus optimization on manifolds. SIAM Journal on Control Optimization, 48(1):56-76, January 2009.

[21] D. Zhou. Quadrotor dynamics and the differential flatness theory based aggressive control. Technical report, Boston University, 2013. http://people.bu.edu/schwager/TechReports/ZhouBUTechReport13.pdf. 